\title{
Sonographic evaluation of hindfoot pain
}

Ibrahim F. Ezz ${ }^{1}$ Msc; Safwat Sayed Araby ${ }^{1}$ MD; Ehab Abdul-Azeim Ahmed ${ }^{1}$ MD; Hany Mohammed Aly ${ }^{1}$ MD

* Corresponding Author:
Ibrahim F. Ezz
dr.ibrahimfawzy@gmail.com

\author{
Received for publication \\ November 21, 2020; Accepted \\ December 30, 2020; Published \\ online December 30, 2020.
}

Copyright 2020 The Authors published by Al-Azhar University, Faculty of Medicine, Cairo, Egypt. All rights reserved. This an open-access article distributed under the legal terms, where it is permissible to download and share the work provided it is properly cited. The work cannot be changed in any way or used commercially.

doi: 10.21608/aimj.2021.50625.1358

${ }^{1}$ Rheumatology and Rehabilitation department, Faculty of medicine, AlAzhar University, Cairo, Egypt

Disclosure: The authors have no
financial interest to declare in
relation to the content of this
article. The Article Processing
$\begin{aligned} & \text { Charge was paid for by the } \\ & \text { authors. } \\ & \text { Authorship: All authors have a } \\ & \text { substantial contribution to the article. }\end{aligned}$

Disclosure: The authors have no inancial interest to declare in article. The Article Processing Charge was paid for by the Authorship: All authors have a substantial contribution to the article.

\begin{abstract}
ABSTRAC.
Background : Hindfoot is frequently injured either duo to trauma, overuse or inflammatory processes. Different diagnoses unfortunately tend to have overlapping clinical signs and symptoms. So, clinicians tend to rely on imaging. Ultrasonography (US) has many benefits for evaluating hindfoot pain.

Aim of the study:to review the prevalence of etiologies in patients with hindfoot pain by musculoskeletal ultrasound.

Patient and methods:This study included one hundred (100) patients complaining of unilateral or bilateral hindfoot pain ranging in age from 16 to 65 years with no sex specification, excluding patients with chronic rheumatological inflammatory diseases, diabetes mellitus, major ankle trauma, previous foot surgery and any foot or lower limp deformity. All patients were subjected to careful history taking, clinical examination, laboratory investigations and $\mathrm{X}$ ray imaging for ankle and foot, giving provisional diagnosis; followed by real-time high resolution ultrasonography for the affected hind foot.

Results: By high-resolution ultrasonography for the affected hindfoot of our patients, 181 different lesions were detected, tendon lesions were the commonest lesions detected, with 63 lesions (35\%) followed by 59 lesions (32\%) for joint \& synovial lesions, 31(17\%) for planter fascia lesions, $12(7 \%)$ for ligament lesions, 9(5\%) for Bursal lesions and 7(4\%) for subcutaneous lesions. Of these all lesions, 29 lesions (16\%) were not clinically suspected.

Conclusion: Ultrasonography is an ideal method for testing patients with hindfoot pain and are highly capable of identifying a wide variety of pathologies, some of which may not be clinically identified.
\end{abstract}

Key Words: Hindfoot; Ultrasound; Ankle pain.

\section{INTRODUCTION}

One of the most important reasons people make an appointment to see a doctor is ankle and foot pain, approximately 20 percent of the population may experience recurrent ankle and foot pain, and over seven million Americans are treated each year for feet-related disorders. As a result, the economic strain of ankle/foot pain costs billions of dollars a year for United States insurance providers. ${ }^{1}$

Via physical examination alone, diagnosing the source of hindfoot pain can be problematic for the clinician. The complicated morphology of the hindfoot means that it can be difficult to localise the symptoms to a particular structure and sadly, multiple diagnoses appear to have overlapping clinical signs and symptoms. Thus, physicians prefer to depend on imaging. ${ }^{2}$

High-resolution ultrasonography has become increasingly important in the assessment of structures around the ankle because its low cost in comparison to CT and MRI, fast, readily available, doesn't have the risk of ionization radiation as in CT and plain radiography, not contraindicated in patients with cardiac pacemakers and metallic implants as in MRI, performed in dynamic manoeuvres and with the advantage of Colour and power Doppler; it has the ability to add essential data about the related vascular structures. Another advantage of ultrasonography is that it is done in real time, which aids the clinicians to identify the site of pain and to compare the affected side with the opposite side. ${ }^{3}$

By this point of view, this work is aimed to review the prevalence of etiologies in patients with hindfoot pain by musculoskeletal ultrasound and to investigate the diagnostic power of ultrasonography for the assessment of painful hindfoot as compared with the clinical findings. 


\section{PATIENT AND MATERIALS}

Patients: A total of one hundred (100) patients complaining of unilateral or bilateral (acute or chronic) hindfoot pain with no sex specification, were involved in this study, and randomly selected from the outpatient clinic of Rheumatology and Rehabilitation department, Al-Hussain University hospital, Cairo, Egypt. The study was conducted during the period from May 2018 to April 2020. Inclusion criteria:

Any patients complaining of unilateral or bilateral hindfoot pain (either acute or chronic) were included in this study.

Exclusion criteria:

Patients less than 16 years and more than 65 years, patients with; chronic rheumatological inflammatory diseases, diabetes mellitus, major ankle or foot trauma causing fracture or dislocation, previous foot surgery, leg length discrepancy, and any patients with foot or lower limp deformity were excluded from this study.

Control group:

Twenty (20) healthy volunteers, age and sex matched, not complaining of any ankle/foot pain or swelling, were added to this study to be examined by ultrasonography for both feet with total forty (40) feet examined as a control group.

Methods:

The study protocol was approved by the local research and ethics committee of Al-Azhar faculty of Medicine (Cairo). All patients and control group signed an informed consent after full explanation of the study protocol.

All patients were subjected to careful history taking, clinical examination, laboratory investigations and $\mathrm{X}$-ray imaging for ankle and foot, giving provisional diagnosis; followed by real-time high resolution ultrasonography for the affected hindfoot.

The ultrasonography was performed (with no extra charge to the patient) by the principal investigator and confirmed by supervisor who are consultant rheumatologist, well trained on musculoskeletal ultrasonography (EULAR certified).

All patients and control group were examined at the musculoskeletal ultrasound unit of rheumatology and rehabilitation department, with commercially available equipment (APLIO 400 Model, Toshiba ultrasound machine) using 8-12 $\mathrm{MHz}$ linear phased array transducer. No preparation was needed and the patient position was changed according to structure examined.

The ultrasonography examination was done in a compartmental manner according to standard scans of European League Against Rheumatism (EULAR). ${ }^{4}$ Each structure examined at both transverses and longitudinal plains, with grey scale, and power Doppler used when pathology seen. For certain structures, both static and dynamic examination were needed.

Anterior compartment: the patient lied in a supine position. Scanning of the ankle was first performed to get a comprehensive view of the tibio-talar joint with separate assessment of the extensor tendons of the ankle (tibialis anterior TA, extensor hallucis longus EHL and extensor digitorum longus EDL) and anterior tibio-fibular ligament.

Lateral compartment: slight inversion of the foot was performed while the patient lied in the supine position to evaluate the lateral collateral ligaments and peroneal tendons.

Medial compartment: the patient was asked to rotate his lower limb laterally in the supine position to assess the deltoid ligament and flexor tendons (tibialis posterior TP, flexor digitorum longus FDL and flexor hallucis longus FHL)

Posterior compartment: The patient was asked to lie in a prone position and rest on his/her toes. The Achilles tendon was examined.

Sole of the foot: the probe was positioned inferiorly in the sagittal plane at the plantar aspect of the foot to evaluate the plantar fascia.

Statistical analysis:

Data were collected, fed to the computer and analyzed using IBM SPSS software package version 20.

\section{RESULTS}

The age of the patients ranged from 17-62 years with a mean age of $39.89 \pm 12.26$ SD. There was no sex specification, the patients was 50 (50\%) Males \& 50(50\%) Females. Patients with unilateral hindfoot pain represented $82 \%$ while the bilateral represented 18\% (table1). 


\begin{tabular}{|c|c|c|c|c|c|}
\hline \multicolumn{2}{|c|}{ Age in years } & \multicolumn{3}{c|}{ Sex } & \multicolumn{2}{c|}{ Lateralization } \\
\hline range & mean & male & female & unilateral & bilateral \\
\hline $17-62$ & $39.89 \pm 12.26$ & $50 \%$ & $50 \%$ & $82 \%$ & $18 \%$ \\
\hline
\end{tabular}

Table 1: distribution of age, sex, and lateralization of the examined patients

There were 24 patients with history of mild trauma and 76 patients with no history of trauma and according to complaint of our patients, $100 \%$ of them were complaining of pain, and $42 \%$ were complaining of swelling (with pain), while tingling represented $3 \%$ of patients complaint.
The lateral ankle compartment was the most common affected compartment (24\%) of cases, followed by the medial compartment (23\%) (table 2).

\begin{tabular}{|c|c|c|c|c|c|c|c|c|c|c|}
\hline \multicolumn{2}{|c|}{ History of trauma } & \multicolumn{4}{|c|}{ Complaint } & \multicolumn{5}{c|}{ Site of pain } \\
\hline Yes & No & Pain & Swelling & Tingling & Anterior & Lateral & Medial & Posterior & Inferior & Whole joint \\
\hline $24 \%$ & $76 \%$ & $100 \%$ & $42 \%$ & $3 \%$ & $12 \%$ & $24 \%$ & $23 \%$ & $12 \%$ & $15 \%$ & $14 \%$ \\
\hline
\end{tabular}

Table 2: patient distribution according to history of trauma, complaint and site of pain

By high-resolution ankle ultrasound, 181 different lesions were detected, tendon lesions were the commonest lesions detected, with 63 lesions (35\%) followed by 59 lesions (32\%) for joint \& synovial lesions, 31(17\%) for planter fascia lesions, 12(7\%) for ligament lesions, 9(5\%) for Bursal lesions and 7(4\%) for subcutaneous lesions (Figure1).

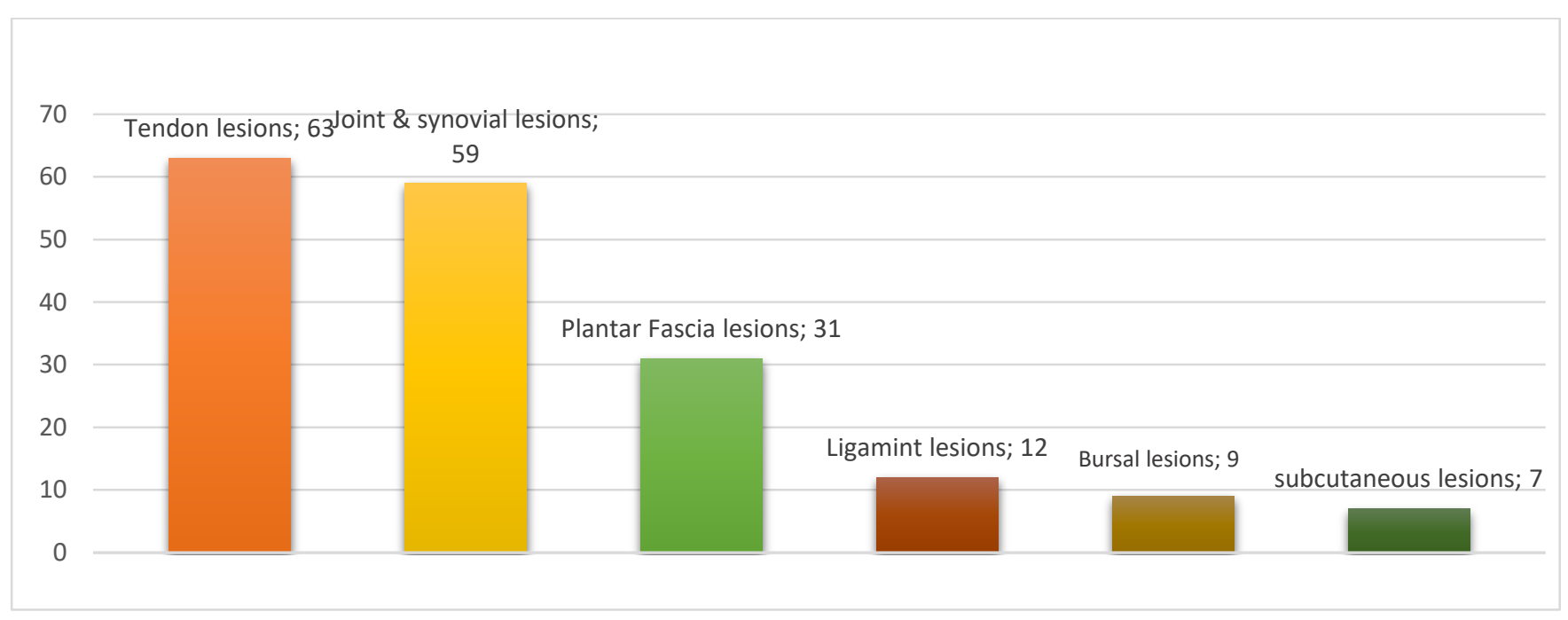

Fig. 1: distribution of different pathological lesions detected by US

For the tendinous lesions (figure 2), 63 different lesions were detected by US in 56 different tendons, tibialis posterior (TP) was the commonest tendon affected (27\%) followed by Achilles then Peroneal (23\% \& $18 \%$ respectively).
Tenosynovitis was the commonest tendinous lesions detected (64\%), followed by partial tear (13\%), enthysophytes (11\%), tendonitis (9\%) and calcification (3\%). 


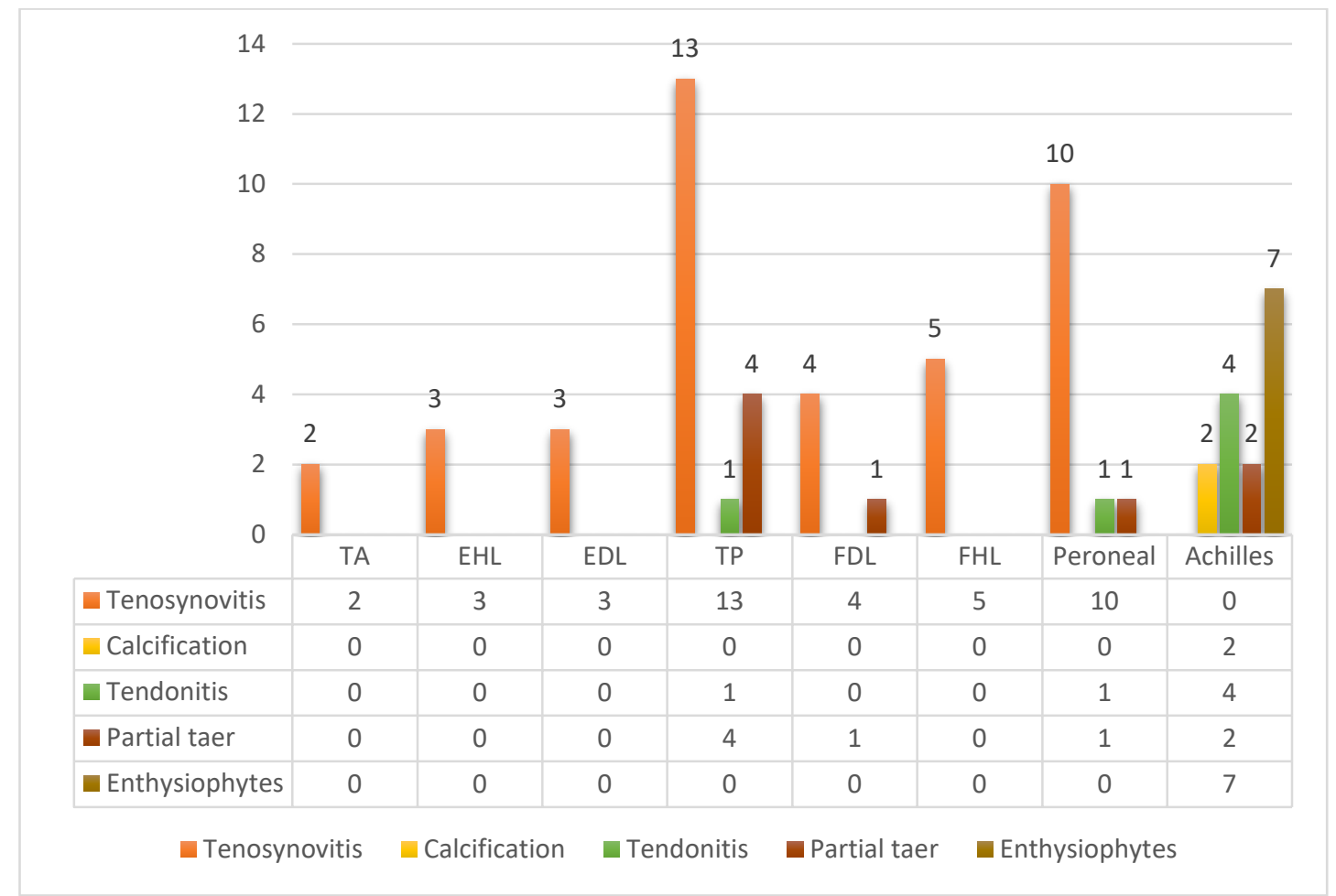

Fig 2: distribution and classification of different tendinous pathology detected by US

For the joint \& synovial lesions, 59 different lesions were detected by US. Tibiotalar joint was the commonest joint affected (56\%), and effusion was the commonest lesion detected (39\%) followed by synovitis (36\%).

Planter fascia lesions represent 31 lesions (17\%) from all lesions detected by US, $64 \%$ of these lesions were Planter fascitis, $23 \%$ were Enthesopathy, $10 \%$ were Heel fat atrophy and 3\% were planter fascia Partial tear.

For the Ligamentous lesions (figure 3), 12 lesions were detected by US, $67 \%$ of these lesions were ligament sprain, while $33 \%$ were partial tear; with the Anterior talofibular ligament was the commonest ligament affected by this lesions (50\%).

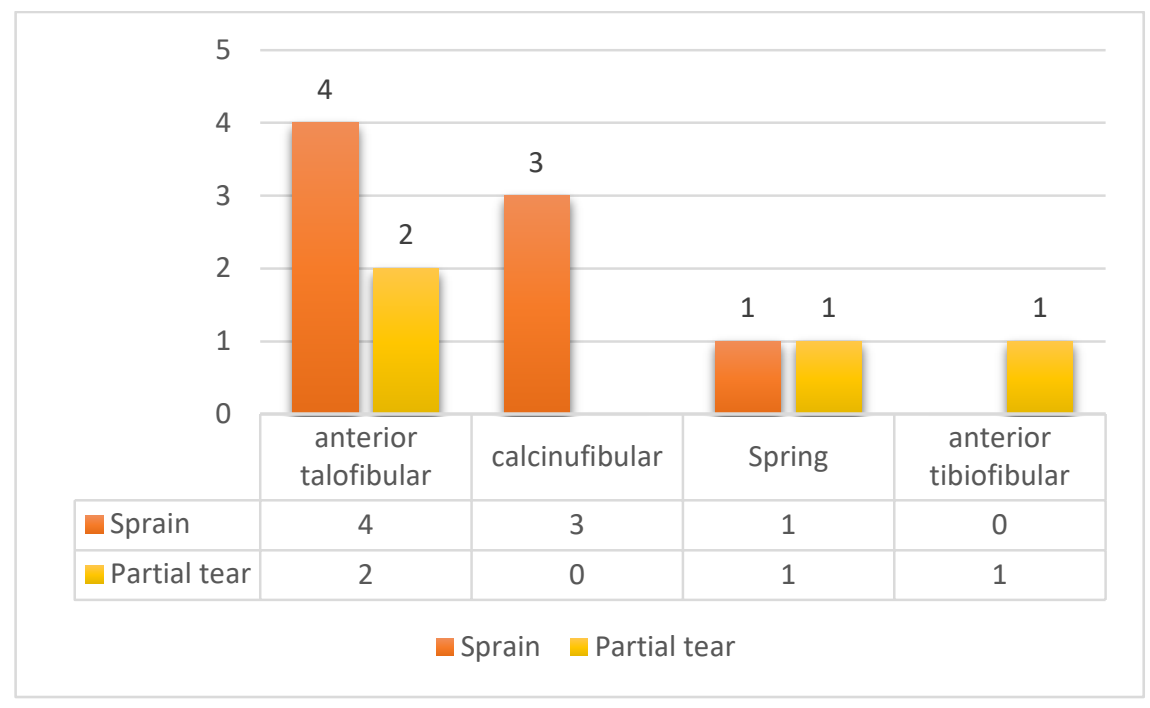

Fig. 3: distribution and classification of different ligamentous pathology detected by US 
Bursal lesions represented nine lesions (5\%) from all lesions detected by US, 5 (56\%) of these lesions were Retrocalcaneal bursitis, 3 (33\%) were Adventitious bursits and one (11\%) were Retroachilles bursitis.

Subcutaneous lesions represented seven lesions (4\%) from all lesions detected by US, three (42\%) of these lesions were fat \& lipoma, two (29\%) were Oedema, and two (29\%) were Xansoma.

Twenty-nine lesions (16\%) from all lesions detected by US were not clinically suspected.

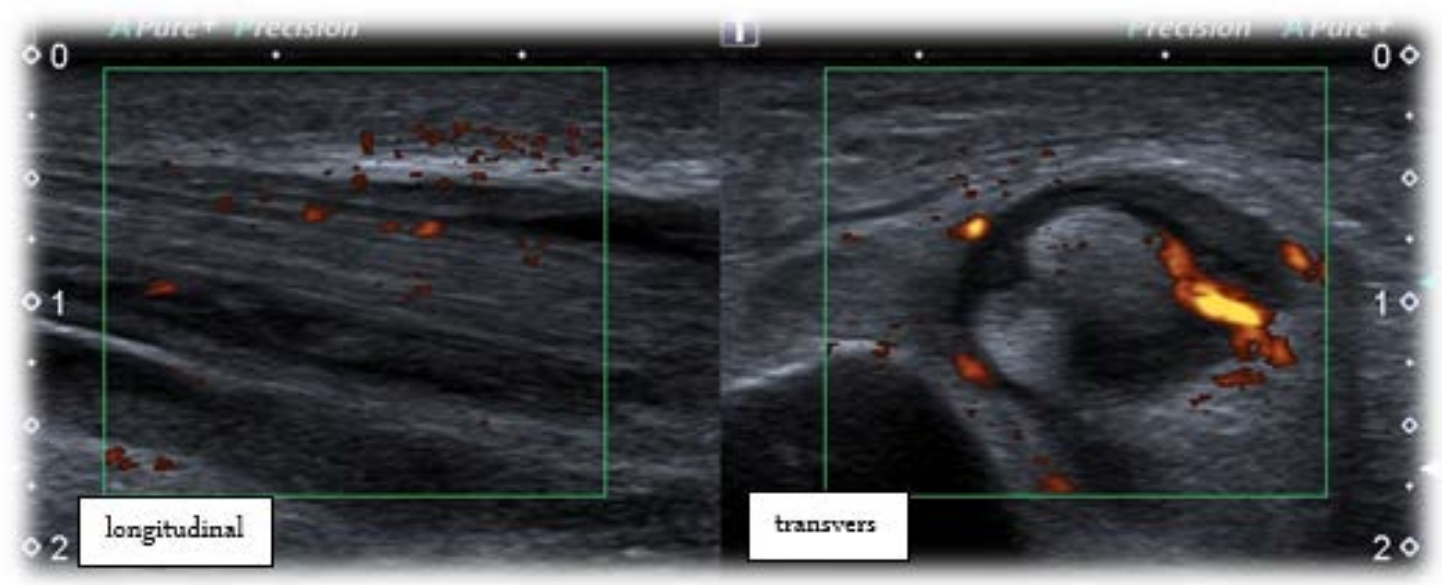

Fig. 4: US transvers \& longitudinal section show peroneal tenosynovitis with Doppler signal
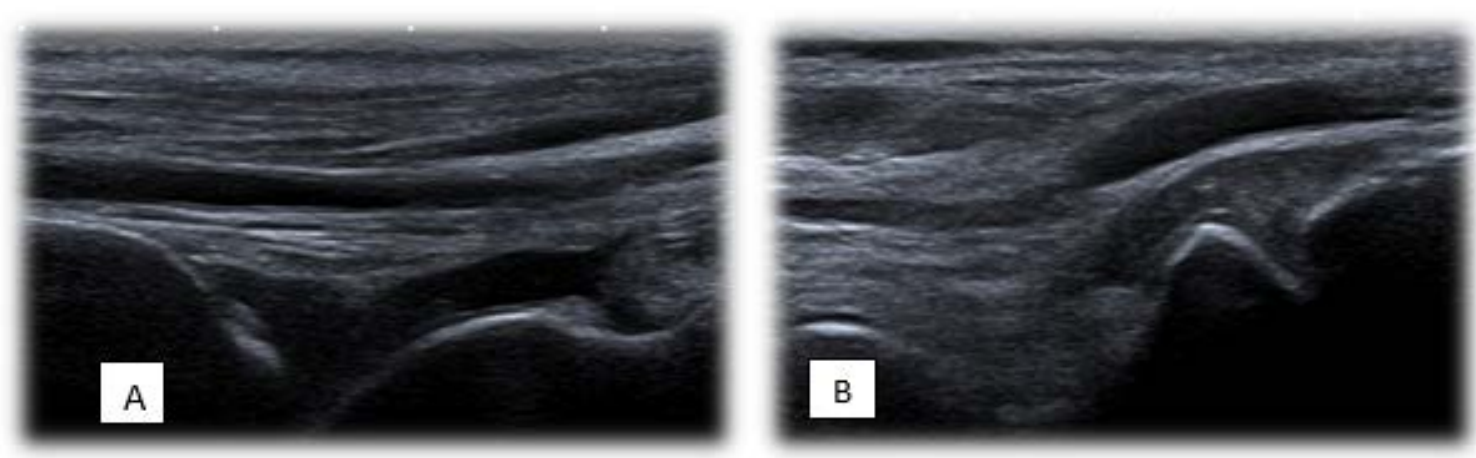

Fig. 5: US longitudinal section show A) tibiotalar joint effusion B: talonavicular synovitis

\section{DISCUSSION}

Most of our patients (80\%) underwent the ultrasound examination within 3 months of the onset of symptoms, whereas $20 \%$ of patients presented with chronic ankle pain ( $>3$ months). This finding is in agreement with the study conducted by Singh et al. ${ }^{5}$ in which $62.8 \%$ of their patients underwent imaging because of acute pain and swelling, while $37.2 \%$ due to chronic symptoms.

There was no sex specification in our study, the patient's sex was 50(50\%) female and 50(50\%) male, patients less than 16 years old and more than 65 years old were excluded from our study with the mean age of the patients was 39.89 years; similar findings were demonstrated by Artul \& Habib. ${ }^{6}$

All of our patients were complaining of pain, while the swelling was the second most presenting symptom (42\%); the same was shown in Muhammad et al.'s study. ${ }^{7}$

The lateral ankle compartment was the most common affected compartment (24\%) of cases, and this is in agreement with the study of Shalaby et al. ${ }^{8}$

The main finding of our study is the huge spectrum of findings that ultrasound was able to visualize; By 
our high-resolution US, 181 different lesions were detected in our patients.

The patients with tendon abnormalities as the cause of pain outnumbered the patients with other abnormalities, representing $35 \%$ of all lesions detected, including tenosynovitis, partial tear, enthysophytes, tendonitis and tendon calcifications. Similar incidence was described in the study of Singh et al. ${ }^{5}$

This is in contrast to the study of Muhammad et al. ${ }^{7}$ in which Synovial pathology was the most prevalent one. This could be explained by the fact that the comorbid conditions such as rheumatoid arthritis and diabetes not excluded in his study but excluded in our study.

Among the detected tendon lesions, Tenosynovitis was the most common lesion representing $64 \%$ of all tendon lesions detected by US. This finding was consistent with the study performed by Shalaby et al. ${ }^{8}$

In our study, US was able to detect abnormalities in all tendons of the four compartments of hindfoot (Anterior, posterior, medial and lateral) with 63 different tendon lesions detected at 56 tendons.

Both Tibialis posterior and Achilles tendons were the commonest tendons affected in our study ( $27 \%$ and $23 \%$ respectively), while peroneal tendon lesions (figure 4) represented 18\%, being the 3rd common tendon affected in our study, and this is in line with the study of Hetta \& Niazi ${ }^{9}$.

In our study, US was able to detect tenosynovitis at only two tibialis anterior tendons and three EDL \& EHL tendons. This agreed with El-Liethy \& $\mathrm{Kamal}^{3}$ who reported that anterior ankle tendons are rarely affected by pathology due to reduced mechanical tension and relative straight tendon coarses.

Joint and synovial lesions including (effusion, synovitis, osteophytes, erosions and ganglion cyst) represented 32\% of all lesions detected by US, being the second common lesions in our study.

Joint effusion was the commonest pathology detected, representing 39\% followed by Synovitis which represented $36 \%$ of joint and synovial lesions (figure 5). Among hindfoot joints, tibiotalar joint was the commonest joint affected by this lesions (56\%). This is in agreement with the study of Muhammad et al. ${ }^{7}$ in which joint disorders accounted for $21 \%$; most of them was tibiotalar effusion.

Ultrasound can differentiate between effusion and synovitis. Static ultrasound examination is able to detect as little as $2 \mathrm{~mL}$ of ankle fluid, versus $1 \mathrm{~mL}$ for ankle MRI. By the aid of dynamic examination, such as dorsiflexion and plantarflexion, ultrasound can be able to detect smaller volumes of fluid. ${ }^{10}$

In our study, three patients were complaining of pain and tingling in the medial ankle, diagnosed clinically as tarsal tunnel syndrome; and ultrasound examination of all of them revealed ganglion cyst (figure 6) compressing the tibial nerve in the tarsal tunnel. This is in agreement with Kawakatsu et al. ${ }^{11}$ who reported that the ganglion and talocalcaneal coalition represented $40 \%-50 \%$ of the cause of tarsal tunnel syndrome

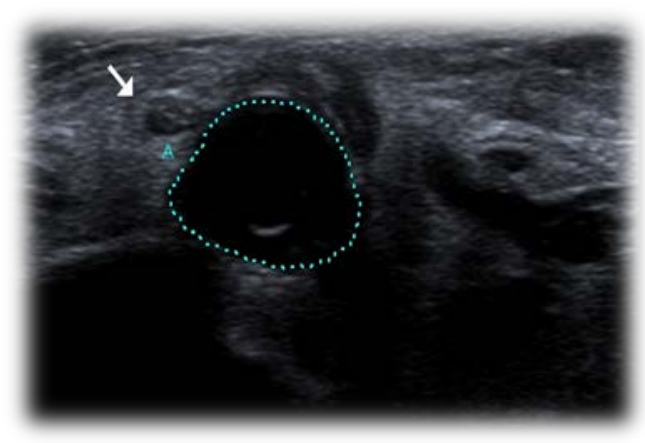

Fig. 6: US longitudinal section of tarsal tunnel show tibial nerve compression by ganglion cyst

In our study, 12 cases of ligamentous injury were diagnosed by US in 11 patients representing $7 \%$ of the whole encountered pathological entities. Anterior talofibular ligament (ATFL) was the most frequently injured ligament representing (50\%) of the whole ligamentous injuries followed by the calcaneo-fibular ligament (CFL) (25\%), in addition to two cases of spring ligament injury and one case of anterior tibiofibular ligament injury (Figure 7) representing $17 \%$ \& $8 \%$ respectively.

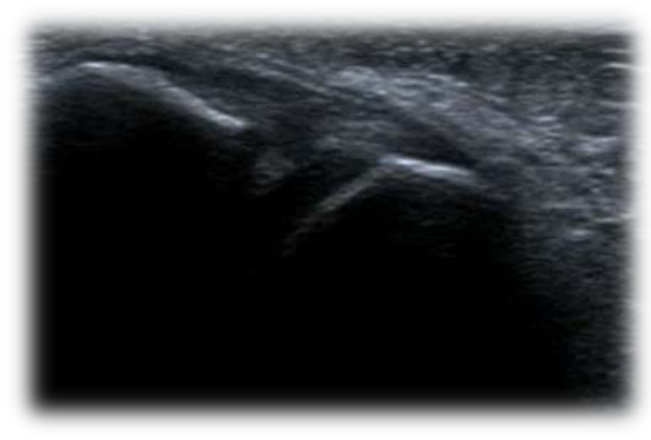

Fig. 7: US longitudinal section show partial tear of Anterior tibiofibular ligament

Our result is in line with the results of El-Liethy \& Kamal ${ }^{3}$ who reported that the lateral collateral ligament complex is affected in $80-90 \%$ of all ankle ligament injuries.

The anterior talofibular ligament is the most often torn ankle ligament since it is the weakest ankle ligament, followed by the calcaneo-fibular ligament, 
while the strongest and least damaged ankle ligament is the deltoid ligament. ${ }^{12}$

Spring ligament abnormalities was detected by US in two patients with tibialis posterior tendon partial tear in our study. This is in line with Harish et al. ${ }^{13}$ who reported that Spring ligament defects are associated with tibialis posterior tendon injuries.

In our study, $15 \%$ of the patients were complaining of inferior hindfoot (heel) pain, with 31 lesions detected at planter fascia (figure 8) by US, representing $17 \%$ of all lesions detected in our study, (64\%) of these lesions were planter fasciitis. This is in agreement with Zubairy et al ${ }^{14}$ who reported that the most frequent cause of inferior hindfoot pain is plantar fasciitis .

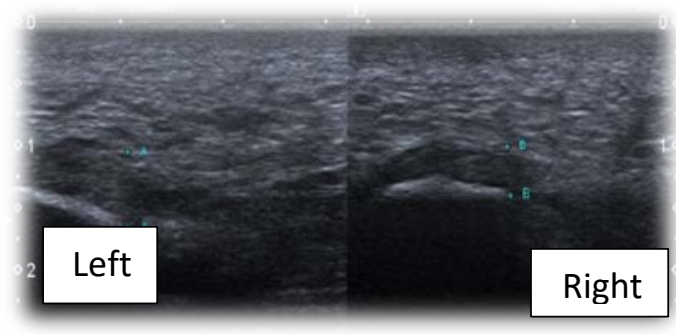

Fig. 8: US longitudinal section show left planter fasciitis

Plantar calcaneal spurs represented $23 \%$ of the planter fascia lesions detected by US in our patients, and all of them accompanied by planter fasciitis. Also plantar calcaneal spurs detected in $9 \%$ of the control group of our study (healthy individuals), and this is in line with Draghi et al. ${ }^{15}$ who reported that it can occur in about $50 \%$ of plantar fasciitis patients, dose not correlate well with symptoms and can be present in asymptomatic peoples as well.

In our study, ultrasound detect heel fat pad atrophy in 3 patients complaining of inferior heel pain, two patients of them give past history of local steroid injection as a treatment for previously diagnosed planter fasciitis.

Local corticosteroid injections are a procedure of plantar fasciitis that is widely used. Fat pad atrophy, plantar fascia rupture, skin depigmentation, and telangiectasia, however are well established drawbacks to local steroid injection.$^{16}$

Bursal lesions represented 9 lesions (5\%) from all lesions detected by US in our study, 5 of these lesions were Retrocalcaneal bursitis, 3 were Adventitious medial malleolar bursits (figure 9), and only 1 was Retroachilles bursitis.

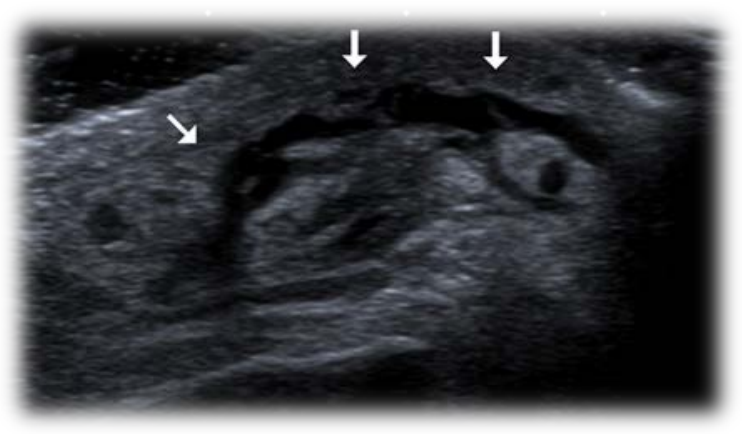

Fig. 9: US transverse section show medial malleolar bursits

This is in line with the study of Hetta \& Niazi ${ }^{9}$ in which 10 bursal lesions were detected during ligamentous and tendinous examination, 8 of them were retrocalcaneal and 2 were adventitious bursa opposite to navicular/medial cuneiform joint.

In our study, US was able to detect bilateral Achilles tendon xanthomas in male, 18 years old patient with family history of familial hypercholesterolemia.This agreed with Artul \& Habib ${ }^{6}$ who detected also Achilles tendon xanthoma by US in one case of their study.

US in our study was able to detect the nature of diffuse ankle swelling in two patients, one of them diagnosed as subcutaneous edema, while the other was cellulitis. This is in line with the ultrasonographic findings in Shalaby et al.'s study ${ }^{8}$ in which both edema and cellulitis were detected among there different pathological lesions.

Three other patients in our study presented by localised ankle swelling, which diagnosed by US as lipomas.

Lipomas may have distinctive US imaging features, including a well-defined ovoid form parallel to the surface of the skin, relative hyperechogenicity and lack of internal blood flow on Doppler assessment. ${ }^{17}$

Post traumatic ankle joint pain was encountered in 2 cases of our study. Only ankle joint mild effusion was detected by US in both patients, While they had talar dome bone marrow contusion and oedema that was missed by US examination and only detected my MRI

Another case was complaining of unilateral chronic anterior ankle pain; in which US detect synovitis of talonavicular joint, while only MRI was able to detect avascular necrosis of navicular bone (Muller Weiss).

This is considered as one of the limitations of US. In this entity, MRI has the upper hand as it correctly recognises early marrow changes, bone viability degradation, location and size of the dead bone fragment. ${ }^{18}$ 
In our study, twenty-nine lesions (16\%) from all lesions cannot detected clinically, while detected only after US examination; most of this lesions was joint \& synovial lesions. This is in line with the study of Singh et al. ${ }^{5}$ in which $18 \%$ of the lesions of his study diagnosed only by US and most of them was joint effusion and synovitis.

\section{CONCLUSION}

Ultrasonography is an ideal method for testing patients with hindfoot pain and are highly capable of identifying a wide variety of pathologies, some of which may not be clinically identifie; As it provides a rapid, dynamic, and cost-effective test, US can be used as the primary imaging investigation for patients having hindfoot pain. However, it has drawbacks when used to assess marrow defects and deep-seated pathologies, for which MRI should be included for diagnosis.

\section{REFERENCES}

1. Zale KE, Klatt MD, Volz KR, et al. Screening Sonography of the Ankle/Foot to Correlate Pain and Pathology. Journal of Diagnostic Medical Sonography. 2016; 32(2), 83-91.

2. Alazzawi S, Sukeik M, King D, et al. Foot \& ankle history and clinical examination: A guide to everyday practice. World J Orthop. 2017; 8: 21-29.

3. El-Liethy $\mathrm{N}$ and Kamal $\mathrm{H}$. High resolution ultrasonography and magnetic resonance imaging in the evaluation of tendino-ligamentous injuries around ankle joint. The Egyptian Journal of Radiology and Nuclear Medicine. 2016; 47(2), 543-555.

4. Richard J, Filippucci E and Grassi W. Sonoanatomy, scanning technique and basic pathology of the ankle and foot. EULAR Online Introductory Ultrasound Course. 2018; module 5.

5. Singh K, Thukral CL, Gupta K, et al. Comparison of high resolution ultrasonography with clinical findings in patients with ankle pain. $J$ Ultrason. 2018; 18: 316-324.

6. Artul S and Habib G. Ultrasound Findings of the Painful Ankle and Foot. Journal of Clinical Imaging Science. 2014; 4(1), 25.

7. Muhammad O, Timothy M and Ian M. Pattern of ultrasonographic findings of disorders of the ankle joint complex in patients presenting with ankle pain at the department of diagnostic imaging, university of Nairobi. Pamj. 2018; 31.116.

8. Shalaby M, Sharara S and Abdelbary M. High resolution ultrasonography in ankle joint pain: Where does it stand? The Egyptian Journal of Radiology and Nuclear Medicine. 2017; 48(3), 645-652.
9. Hetta W and Niazi G. Concordance of US and MRI for diagnosis of ligamentous and tendinous injuries around the ankle. The Egyptian Journal of Radiology and Nuclear Medicine. 2018; 49(1), 131-137.

10. Bruyn G, Iagnocco A, Naredo E, et al. OMERACT Definitions for Ultrasonographic Pathology and Elementary Lesions Of Rheumatic Disorders Fifteen Years On. jrheum. 2019; 181095.

11. Kawakatsu M, Ishiko $\mathrm{T}$ and Sumiya M. Tarsal Tunnel Syndrome Due To Three Different Types of Ganglion During a 12-Year Period: A Case Report. The Journal of Foot and Ankle Surgery. 2017; 56(2), 379-384

12. Cheng Y, Cai $Y$ and Wang Y. Value of ultrasonography for detecting chronic injury of the lateral ligaments of the ankle joint compared with ultrasonography findings. Br J Radiol. 2014; 87:1-6.

13. Harish S, Kumbhare D, O’Neill J, et al. Comparison of sonography and magnetic resonance imaging for spring ligament abnormalities: preliminary study. $J$ Ultrasound Med. 2008; 27: 1145-1152.

14. Zubairy A, Khan S and Bhatty U. Managing the patient with heel pain. British Journal of Hospital Medicin. 2019; 80(4), 196-200.

15. Draghi F, Gitto S, Bortolotto C, et al. Imaging of plantar fascia disorders: findings on plain radiography, ultrasound and magnetic resonance imaging. Insights into Imaging. 2016; 8(1), 69-78.

16. Rosenbaum A, DiPreta J and Misener D. Plantar Heel Pain. Medical Clinics of North America. 2014; 98(2), 339-352.

17. Fessell DP and Jacobson JA. Ultrasound of the hindfoot and midfoot. Radiol Clin North Am. 2008; 46(6):1027-43.

18. Beard NM and Gousse RP. Current Ultrasound Application in the Foot and Ankle. Orthopedic Clinics of North America. 2018; 49(1), 109-121. 